\title{
Cholesterol Flip-Flop in Heterogeneous Membranes
}

\author{
Ruo-Xu Gu, ${ }^{\dagger}$ Svetlana Baoukina, and D. Peter Tieleman*i] \\ Centre for Molecular Simulation and Department of Biological Sciences, University of Calgary, 2500 University Drive, N.W., \\ Calgary, Alberta T2N 1N4, Canada
}

\section{Supporting Information}

\begin{abstract}
Cholesterol is the most abundant molecule in the plasma membrane of mammals. Its distribution across the two membrane leaflets is critical for understanding how cells work. Cholesterol trans-bilayer motion (flip-flop) is a key process influencing its distribution in membranes. Despite extensive investigations, the rate of cholesterol flip-flop and its dependence on the lateral heterogeneity of membranes remain uncertain. In this work, we used atomistic molecular dynamics simulations to sample spontaneous cholesterol flip-flop events in a DPPC:DOPC:cholesterol mixture with heterogeneous lateral distribution of lipids. In addition to an overall flip-flop rate at the time scale of submilliseconds, we identified a significant impact of local environment on flip-flop rate. We discuss the atomistic details of the flip-flop events observed in our simulations.
\end{abstract}

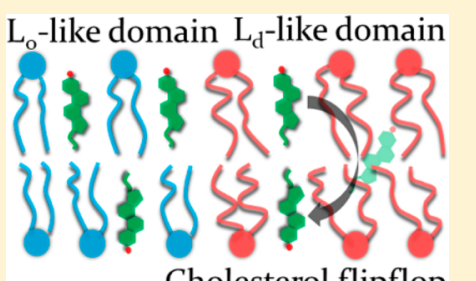

Cholesterol flipflop

\section{INTRODUCTION}

Lipids are distributed unevenly across the two leaflets of the plasma membrane: sphingomyelin and phosphatidylcholine (PC) are mainly located in the extracellular leaflet, whereas phosphatidylethanolamine (PE), phosphatidylserine (PS), and phosphatidylinositol (PI) are confined largely to the cytoplasmic leaflet. $^{1-3}$ The uneven lipid distribution has clear biological functions. For instance, the negative charge associated with PS at the cytosolic side is a mechanism for protein sorting to the plasma membrane; exposure of PS to the extracellular side mediated by scramblases is a signal for blood coagulation and removal of apoptotic cells. ${ }^{3,4}$ The asymmetric distribution of lipids is maintained by lipid trans-bilayer motion (flip-flop). In the case of phospholipids, this process is facilitated by ATPdependent flippases (e.g., P-type ATPase) and floppases (e.g., ATP binding cassette transporters). ${ }^{1-3,5}$

Cholesterol is the most abundant molecule in the plasma membranes of mammals. However, in contrast to phospholipids, experiments based on different techniques revealed controversial results regarding cholesterol distribution across the two leaflets, with the proportion of cholesterol in the outer leaflet ranging from $\sim 20 \%$ to $\sim 90 \%$. $^{6}$ In addition, the physiological significance of cholesterol distribution remains largely unknown, although recent research suggests cholesterol is involved in the Wnt signaling pathway and regulates various cellular processes. Cholesterol flip-flop is a critical process that regulates cholesterol trans-bilayer distribution and modulates the organization of cell membranes. Unlike phospholipids with a slow spontaneous flip-flop on physiological time scales (halftime of hours ${ }^{8-10}$ ), trans-bilayer motion of cholesterol is much faster, ${ }^{11-17}$ due to their small hydrophilic headgroups, and does not require ATP. Fast cholesterol flip-flop balances the area of two leaflets, thus relaxing the stresses in the bilayers. ${ }^{12,18}$

Despite extensive studies, cholesterol flip-flop rates remain uncertain. The time scales obtained by experiments ${ }^{12,13,15,19}$ and molecular dynamics (MD) simulations ${ }^{11,20-24}$ range from sub- milliseconds to minutes. In most current simulations, estimates at the atomistic level are based on free energy calculation, whereas flip-flop rates evaluated by counting spontaneous flipflop events have been only obtained at the coarse-grained level. In MD simulations, cholesterol flip-flop is faster in bilayers with unsaturated lipids $^{24,25}$ and lower cholesterol concentration, ${ }^{11,20}$ suggesting a significant influence of the membrane composition. The majority of these simulations were conducted in binary mixtures of cholesterol and phospholipids (e.g., DPPC, POPC, and DAPC) that have homogeneous lateral distribution of components. However, increasing evidence suggests that plasma membranes have a heterogeneous lateral organization. ${ }^{26-29}$ In this work, we describe atomistic simulations of model membranes with coexisting domains to (1) estimate flip-flop rates based on spontaneous flip-flop events at the atomistic level; (2) assess the dependence of cholesterol flip-flop rate on the local environment in a heterogeneous membrane; and (3) characterize in atomic detail the cholesterol flip-flop process.

\section{METHODS}

Molecular Dynamics Simulations. We conducted molecular dynamics (MD) simulations of a ternary mixture (1,2dipalmitoyl-sn-glycero-3-phosphocholine (DPPC):1,2-dioleoylsn-glycero-3-phosphocholine (DOPC):cholesterol = 0.35:0.35:0.3, 1152 lipids and 57600 TIP3P water molecules ${ }^{30}$ in total $)$ at $290 \mathrm{~K}(9 \mu \mathrm{s})$ and $310 \mathrm{~K}(5 \mu \mathrm{s})$, respectively, using the GROMACS4.6.5 package, $^{31}$ the Slipids force field, ${ }^{32-34}$ and an integration time step of $2 \mathrm{fs}$. A cutoff value of $1.0 \mathrm{~nm}$ was used to calculate the van der Waals and electrostatic interactions. The

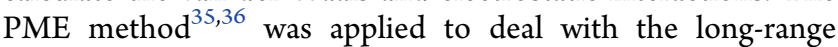
electrostatic interactions with a tolerance of $10^{-5}$, Fourier grid spacing of $0.12 \mathrm{~nm}$, and an interpolation order of 4 for the Ewald mesh. We used the $v$-rescale algorithm ${ }^{37}$ and a relaxation time of

Received: September 14, 2018

Published: January 11, 2019 
1 ps to maintain the temperature of the lipids and solvents at desired values separately. The pressure of the simulation system was maintained at 1 bar using the Parrinello-Rahman barostat $^{38,39}$ with an isothermal compressibility of $4.5 \times 10^{-5}$ $\mathrm{bar}^{-1}$, semi-isotropic pressure coupling method (the $z$ direction independent from the $x$ and $y$ directions), and a relaxation time of 3 ps. Long-range dispersion corrections for pressure and energy were applied. The LINCS algorithm ${ }^{40}$ was used to constrain all covalent bonds in lipids. The initial conformation of the bilayer was constructed using a custom-made software. ${ }^{41,42}$ The initial conformation represents a random mixture; lateral heterogeneity develops over the course of simulation.

Data Analysis. To characterize cholesterol flip-flop, we monitored translocation of cholesterol between the leaflets over the course of the simulation. A cholesterol molecule was considered to be located in one leaflet if its headgroup (the oxygen atom) was at least $0.9 \mathrm{~nm}$ away from the bilayer center along the bilayer normal. This cutoff value was based on the cholesterol oxygen atom distribution along the bilayer normal. A successful cholesterol flip-flop event was defined as a translocation from one leaflet to another, followed by lingering in the second leaflet for at least $10 \mathrm{~ns}$. An aborted flip-flop attempt was defined if cholesterol left one leaflet and then came back, followed by residing in the same leaflet for at least $10 \mathrm{~ns}$.

To identify and quantify lateral heterogeneity of the membranes, we calculated average order parameter maps projected on the membrane plane. The order parameter $\left(\left|S_{\mathrm{cd}}\right|\right)$ of lipid tails was found using the following equation:

$$
\left|S_{\mathrm{cd}}\right|=\left|\frac{\left\langle\left(3 \cos ^{2} \theta-1\right)\right\rangle}{2}\right|
$$

where $\theta$ was the angle between $\mathrm{C}-\mathrm{H}$ vector and the bilayer normal. The last 0.06 and $0.30 \mu$ s trajectories were used to calculate order parameter maps at 310 and $290 \mathrm{~K}$, respectively; different time intervals for averaging reflect different lipid mobilities.

The enrichment value was defined to quantify heterogeneity of the local environment. The enrichment value of lipid A in the local environment of cholesterol, $E_{(\mathrm{AlCHOL})}$, was defined as a ratio of its local to its bulk concentrations:

$$
E_{(\mathrm{AlCHOL})}=\frac{[\mathrm{A}]_{\text {local }}}{[\mathrm{A}]_{\text {bulk }}}
$$

where $[\mathrm{A}]_{\text {local }}$ was the local molar concentration of lipid A within a cutoff distance from cholesterol and $[\mathrm{A}]_{\text {bulk }}$ was its bulk concentration. Here, the distances were calculated between molecular centers of mass (COM) projected on the membrane plane; we used the cutoff value of $1.05 \mathrm{~nm}$, corresponding to the position of the first local minimum of the lipid-cholesterol radial distribution function, thus giving the first coordination shell of lipids around the central cholesterol. An enrichment value above 1 indicates an increased concentration of lipid $A$ in the local environment of cholesterol, while a value below 1 indicates depletion. The enrichment values of DOPC and DPPC in the local environment of successfully flip-flopped cholesterol molecules were calculated for the $10 \mathrm{~ns}(310 \mathrm{~K})$ and $50 \mathrm{~ns}$ (290 K) trajectories before and after each flip-flop event separately. The enrichment value of DOPC in the local environment of DPPC was also calculated (using a cutoff of $1.30 \mathrm{~nm}$ ) to quantitatively compare the lateral heterogeneity at 310 and 290 $\mathrm{K}$. We also calculated the overlap of DOPC enrichment values of the two leaflets. The 2D histograms of these enrichment values show the correlation of local heterogeneity of the two leaflets. Results were compared for the aborted and successful flip-flop attempts and the whole bilayer. Calculation for flip-flopped cholesterol was based on the frames in which cholesterol resided in neither leaflet, and the COMs of cholesterol were used as central points to define the local environment. Calculation for the whole bilayer was based on a grid layout along the membrane plane with a grid spacing of $0.5 \mathrm{~nm}$, using the grid points as central points.

The overall flip-flop rate was calculated by dividing the number of flip-flop events by the total number of cholesterol molecules in the simulation box. To assess the dependence of flip-flop rate on the bilayer lateral heterogeneity, we calculated the flip-flop rate as a function of the enrichment value, by dividing the number of flip-flop events with a specific range of enrichment values by the total number of cholesterol molecules with the same range of enrichment values.

To evaluate cholesterol orientation in the membranes, we used the hidden Markov model (HMM) described in Sodt et al.'s work ${ }^{43}$ to assign a specific state (Lo- or Ld-like state) to each lipid, and then calculated the distribution of cholesterol tilt angles in the Lo- and Ld-like states separately. Briefly, the composition of the five nearest lipids of each lipid in the membrane, as well as the lipid itself, was considered as the emission signal and used as input to train the HMM model, and the most probable state of each lipid was determined. We also calculated the average order parameters of phospholipids in the local environment of cholesterol as a function of their tilt angles for the Lo- and Ld-like domains, respectively. Results are compared for all cholesterol and the flip-flopped cholesterol (including both the aborted and successful flip-flop attempts) during their translocation process.

Statistics of flip-flop events discussed below were based on trajectories of the last $4 \mu \mathrm{s}$ at $310 \mathrm{~K}$ and the last $6 \mu \mathrm{s}$ at $290 \mathrm{~K}$ (which allows sufficient time for convergence of lipid segregation, see Supporting Information Figure S1) unless otherwise specified. The trajectories were divided in blocks of 1 $\mu \mathrm{s}(310 \mathrm{~K})$ and $2 \mu \mathrm{s}(290 \mathrm{~K})$, and the standard error of the mean shown in this work is calculated based on the average of each block. We used Python and MDAnalysis ${ }^{44}$ to write the script to do the above analysis.

\section{RESULTS}

Lateral Heterogeneity of Bilayers. Enrichment values of DOPC in the local environment of DPPC (0.93 and 0.82 at 310 and $290 \mathrm{~K}$, respectively) suggest a heterogeneous lateral arrangement of lipids at both temperatures, consistent with coexisting domains/clusters with different order parameters (I $S_{\text {cd }}$ l of $\sim 0.35$ and 0.20 ) shown in Figure 1. As expected, comparison of the density maps and order parameter maps suggests enrichment of DPPC in the more ordered domains/ clusters (Lo-like) and DOPC in the less ordered domains/ clusters (Ld-like; see Figure 1). HMM analysis shows that lipid ratios (DPPC:DOPC:CHOL) in the Lo- and Ld-like domains at $310 \mathrm{~K}$ are 0.48:0.15:0.37 and 0.20:0.60:0.20, respectively. Corresponding values at $290 \mathrm{~K}$ are 0.57:0.09:0.35 and $0.16: 0.59: 0.25$. The lateral heterogeneity at $310 \mathrm{~K}$ is likely a manifestation of composition fluctuations in the Ld phase, ${ }^{45}$ while at $290 \mathrm{~K}$ it represents coexisting Lo and Ld phases, based on the dynamics and properties of the clusters. For instance, the clusters at $290 \mathrm{~K}$ exist stably for $\sim 1 \mu \mathrm{s}$, whereas the clusters at $310 \mathrm{~K}$ disassemble within $\sim 0.1-0.2 \mu \mathrm{s}$; the average order parameters of DPPC in the Lo- and Ld-like clusters show larger 


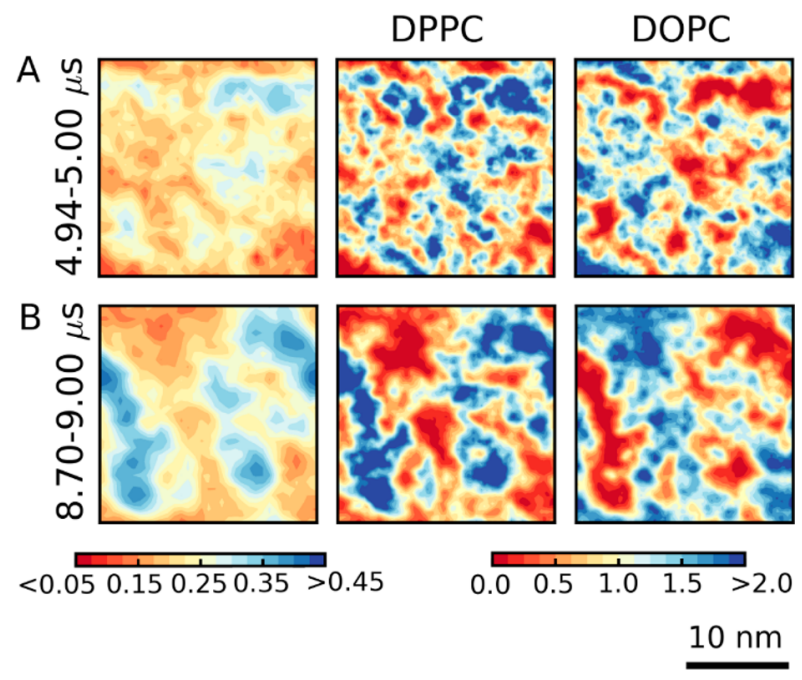

Figure 1. Lateral heterogeneity of membranes in MD simulations. The order parameter maps and lipid density maps are calculated for the last (A) $0.06 \mu \mathrm{s}$ and (B) $0.30 \mu \mathrm{s}$ trajectories of the 310 and $290 \mathrm{~K}$ simulations, respectively. The density maps show relative densities which are normalized to the average lipid density in the simulation box.

differences at $290 \mathrm{~K}$ than at $310 \mathrm{~K}(0.345$ and 0.270 vs 0.278 and 0.230 ), suggesting that Lo-like clusters at $290 \mathrm{~K}$ likely represent Lo phase. In this study we only focus on cholesterol flip-flop.

Cholesterol Orientation in Bilayers. Cholesterol tilt angle distributions (relative to the bilayer normal) and the averages are shown in Figure 2A. Smaller tilt angles at lower temperature
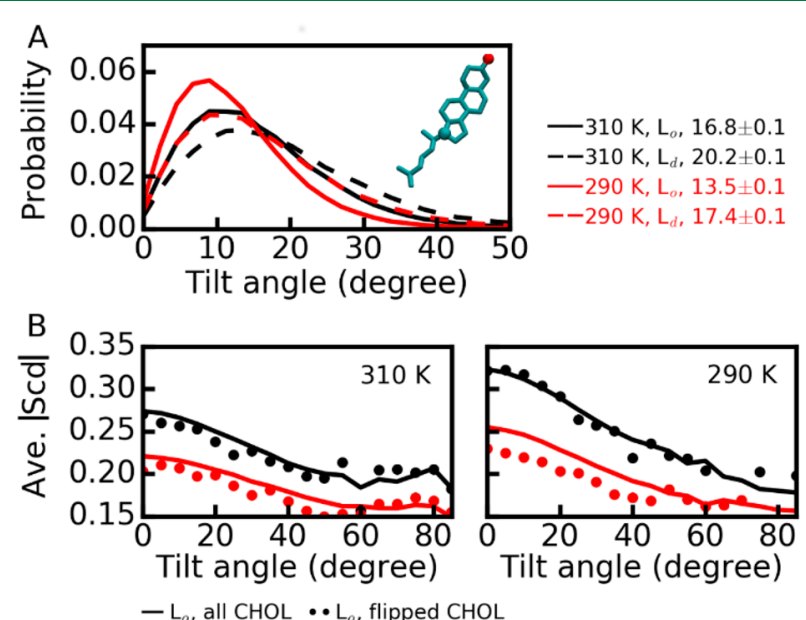

$-\mathrm{L}_{o}$, all $\mathrm{CHOL} \cdots \mathrm{L}_{o}$, flipped $\mathrm{CHOL}$

Figure 2. Cholesterol orientation in bilayers. (A) Distributions of cholesterol tilt angle in the Lo- and Ld-like domains. Cholesterol tilt angle is defined as the angle between the axis connecting atoms $\mathrm{O} 3$ and C17 (shown in spheres in panel A) and the bilayer normal. (B) Average $\left|S_{\text {cd }}\right|$ in the local environment of cholesterol as a function of cholesterol tilt angle in the Lo- and Ld-like domains, respectively. Results are calculated for all cholesterol and the flip-flopped cholesterol (including both aborted and successful attempts), respectively.

and in the more ordered (Lo-like) domains suggest the impact of tightly packed lipid tails on cholesterol orientation. We note that the average tilt angles in the two phases are smaller than those in DPPC, DOPC:cholesterol binary mixtures at $323 \mathrm{~K}$ in ref 46 , respectively $\left(\sim 14-17^{\circ}\right.$ vs $\sim 19^{\circ}$ for Lo-like domains ${ }^{46}$ ), likely due to lower temperatures in this work; other factors, such as differences in the bilayer/domain composition are expected to play a role. We also found correlation between the cholesterol tilt angle and the average order parameter $\left(I S_{\mathrm{cd}} \mid\right)$ of its local environment, for both the Lo- and Ld-like domains (Figure 2B,C), consistent with Aittoniemi et al.'s work. ${ }^{46}$ The average I $S_{\mathrm{cd}}$ l values in the local environment of flipped cholesterol are slightly smaller than that of all cholesterol molecules, particularly for the Ld-like domains at $290 \mathrm{~K}$ (Figure 2B,C). All of these data suggest that cholesterol orientation and flip-flop are sensitive to lipid packing in the local environment.

Cholesterol Flip-Flop Rate. All cholesterol flip-flop events found in the trajectories are listed in Tables S1 and S2. The flipflop rates in this work are at the sub-millisecond time scale (Table 1). ${ }^{11,20-24}$ Lowering the temperature from 310 to $290 \mathrm{~K}$

Table 1. Cholesterol Flip-Flop in Simulations ${ }^{a}$

$\begin{array}{ccc}\text { temp }(\mathrm{K}) & \text { no. of events } & \text { flip-flop rate }\left(\times 10^{4} \mathrm{~s}^{-1}\right) \\ 310 & 138 & 10.0 \pm 1.0 \\ 290 & 20 & 1.0 \pm 0.2\end{array}$

${ }^{a}$ Numbers are based on the last $4 \mu \mathrm{s}(310 \mathrm{~K})$ and the last $6 \mu \mathrm{s}(290$ K) of each trajectory.

slows down flip-flop rates significantly, by approximately 1 order of magnitude $\left((10.0 \pm 1.0) \times 10^{4}\right.$ and $(1.0 \pm 0.2) \times 10^{4} \mathrm{~s}^{-1}$, Table 1). The majority of the flip-flop events take less than $50 \mathrm{~ns}$ at both temperatures, with the events at $310 \mathrm{~K}$ taking less time on average (Tables S1 and S2).

The local environment of flip-flopped cholesterol is enriched in DOPC and depleted of DPPC, compared with that of all cholesterol in the simulation box (see the averages and histograms of lipid enrichment values in Table S3, Figure 3A, and Figure S2). This suggests a preference of the flip-flop event for a local environment with loosely packed lipid tails. For instance, the averages of DOPC enrichment values at $290 \mathrm{~K}$ are 1.5-1.6 for flip-flopped cholesterol, significantly larger than the corresponding value for all cholesterol (0.96, Table S3). The distributions of these enrichment values for flip-flopped cholesterol are shifted to larger values (Figure 3A). Similarly, notably smaller DPPC enrichment values are found for the flipflopped cholesterol (Figure S2 and Table S3). As mentioned previously, we calculated the enrichment values of phospholipids around flip-flopped cholesterol molecules for the leaflet they reside in before and after the flip-flop event separately (Figure 3A, Figure S2, and Tables S1-S3). The averages and histograms of enrichment values before and after the flip-flop events are consistent. This result indicates that cholesterol prefers a less ordered local environment for moving both out of and into a leaflet. In other words, a cholesterol flip-flop event is more likely if the DOPC-enriched environments are in register in the two leaflets. Figure S3 shows that the majority of both aborted and successful attempts happened in the local environment with overlapping disordered clusters. However, the ratio of uncoupled local environment is higher for the aborted attempts than for the successful events, suggesting that the incompatible ordering of the leaflets is one reason for unsuccessful attempts.

Figure 3B indicates a high correlation between flip-flop rates and the local DOPC enrichment values. At $310 \mathrm{~K}$, as the local DOPC enrichment values vary from smaller than 0.4 to larger than 1.6, the flip-flop rates increase by approximately 7 times, with the values ranging from $\sim 3 \times 10^{4}$ to $\sim 20 \times 10^{4} \mathrm{~s}^{-1}$ (Figure $3 \mathrm{~B})$. A similar trend and range of values are found as the local 

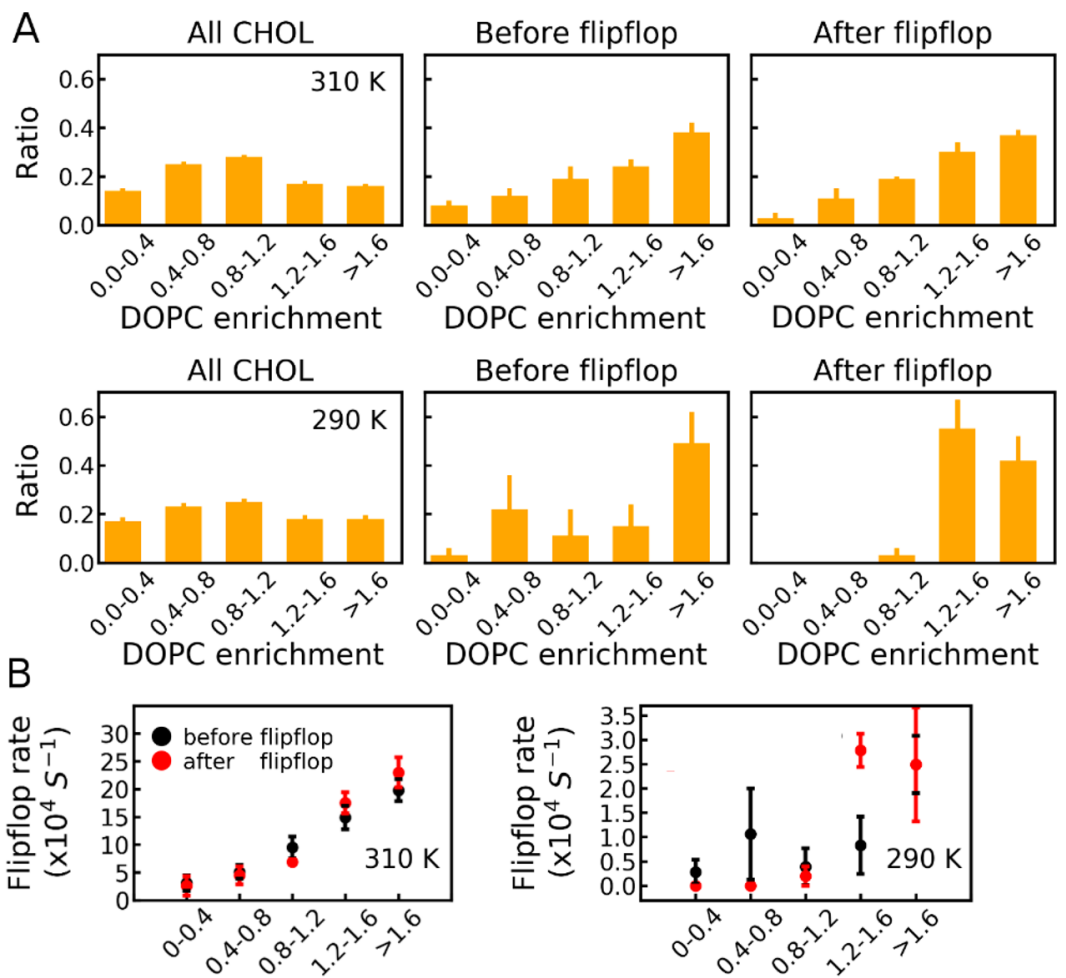

DOPC enrichment

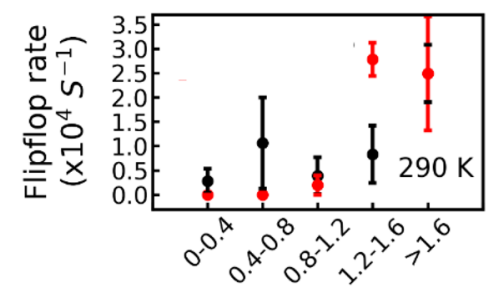

DOPC enrichment

Figure 3. Dependence of cholesterol flip-flop on the local environment. (A) Histograms of DOPC enrichment values in the local environment of all cholesterol in the simulation system, and flip-flopped cholesterol (before and after the flip-flop event), respectively. (B) Cholesterol flip-flop rate as a function of DOPC enrichment value (before and after the flip-flop event) of the local environment.
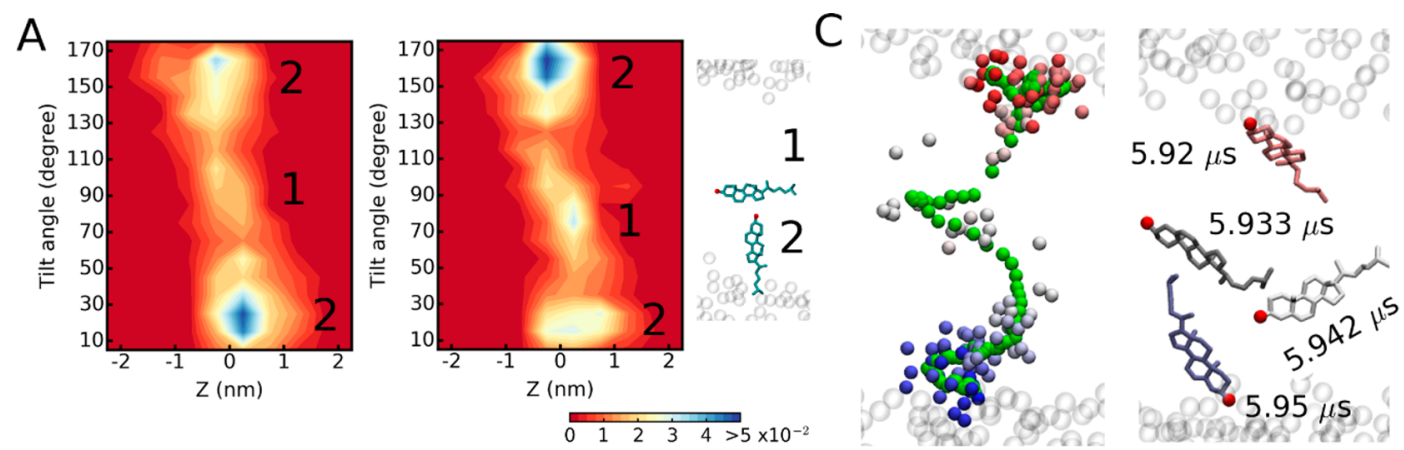

\section{B}
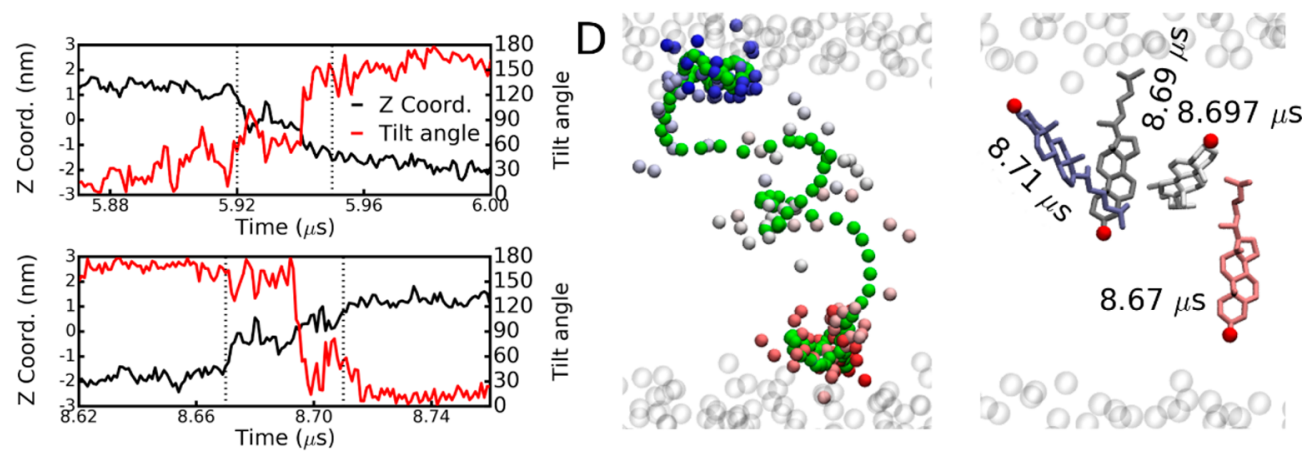

Figure 4. Atomistic details of cholesterol flip-flop pathway. (A) Two-dimensional histogram of cholesterol headgroup position and tilt angle during the flip-flop process (left, $310 \mathrm{~K}$; right, $290 \mathrm{~K}$ ), with the regions corresponding to the two main cholesterol orientations in the bilayer center labeled by numbers (1 and 2). (B) Headgroup position and tilt angle as a function of simulation time of representative flip-flop events at 290 K (top, residue 470; bottom, residue 1014). Start and end times of flip-flop events are labeled by dashed lines. Trace of headgroup (left panel) and example conformations (right panel) of cholesterol during the flip-flop process of residues (C) 470 and (D) 1014 at $290 \mathrm{~K}$. Positions of cholesterol headgroup during the flipflop process are colored from red to blue as a function of time. Green spheres are smoothed traces of headgroups, and the white spheres are lipid phosphorus atoms. 
DPPC enrichment values decrease (Figure S2). The same data analyses reveal flip-flop rates ranging from 0 to $\sim 2.5 \times 10^{4} \mathrm{~s}^{-1}$ at $290 \mathrm{~K}$ as the local DOPC enrichment values increase, indicating the same influence of local environment on flip-flop rate at both temperatures (Figure 3B and Figure S2). A flip-flop rate of 0 simply means that, in DOPC-depleted environment (enrichment value $<0.4$, Figure 3B) at $290 \mathrm{~K}$, cholesterol flip-flop is so slow that the simulation time is not enough to sample flip-flop events. The majority of the flip-flop events found in the trajectory occurs in a DOPC-enriched environment (enrichment value $>1.2$ ). We conclude that the difference in flip-flop rate with respect to the local environment at $290 \mathrm{~K}$ must be larger than at $310 \mathrm{~K}$, but it is not possible to quantify this difference on the time scale of several microseconds. These data indicate that lipids in the first shell of cholesterol have a nonnegligible impact on cholesterol flip-flop rate, regardless of the phase state of the bilayers.

Atomic Details of the Cholesterol Flip-Flop Process. Two-dimensional histograms (Figure 4A) of the headgroup position and tilt angle of cholesterol during the translocation process show a wide range of cholesterol orientations as cholesterol moves from one leaflet to another. The cholesterol tilt angle varies all the way between parallel and perpendicular to the bilayer normal, with two peaks in the distribution at $\sim 150-$ $180^{\circ} / 0-30^{\circ}$ and $\sim 90^{\circ}$, consistent with Bennett and Tieleman's simulation. ${ }^{20}$ Panels B-D of Figure 4 show the cholesterol headgroup position and orientation as a function of simulation time, as well as snapshots of two example flip-flop events, in which the cholesterol assumes different orientations when its headgroup lingers in the bilayer center. Panels B-D of Figure 4 reveal two parameters in the cholesterol flip-flop process: (1) adjustment of the cholesterol orientation and (2) translocation of the headgroup to another leaflet. Parisio and co-workers calculated a two-dimensional free energy landscape of cholesterol flip-flop as a function of tilt angle and headgroup position using an implicit membrane model and constructed a kinetic model for the flip-flop process. ${ }^{21}$ They categorize the flipflop according to two pathways based on how the hydrophilic headgroup moves to the bilayer center: by rotation or translation. In pathway 1 , the cholesterol headgroup moves to the bilayer center by rotation of the molecular axis before it crosses the bilayer midplane. In pathway 2, a cholesterol translocates to the other leaflet first before it reverts the headgroup-tail orientation. When the cholesterol headgroup resides in a bilayer center, its molecular axis is perpendicular or parallel to the membrane normal for pathways 1 and 2, respectively. The observed flip-flop events in our simulations (Figure 4B) agree with this model, with the added detail of allatom lipids.

\section{DISCUSSION}

Previous atomistic free energy calculations gave estimates of flipflop rates between $\sim 0.1 \times 10^{4}$ and $800 \times 10^{4} \mathrm{~s}^{-1} ;^{20,24,47}$ our results in Table 1 are qualitatively consistent with these values. The wide range of flip-flop rates is due to different bilayer compositions in these simulations and possibly due to differences in force fields, effectively resulting in different bilayer properties and densities. Comparing the most similar, although more ordered, system in the literature (DPPC:cholesterol = 0.8:0.2 at $323 \mathrm{~K}^{47}$ ) and this work (DPPC:DOPC:cholesterol = 0.35:0.35:0.3 at $310 \mathrm{~K})$, the cholesterol flip-flop rate is higher in our simulations $\left(0.12 \times 10^{3}\right.$ to $0.81 \times 10^{4}$ vs $\left.10 \times 10^{4} \mathrm{~s}^{-1}\right)$, both within the sub-millisecond time scale. Despite different setups between the simulations in our work and in the literature (i.e., different bilayer compositions, e.g., DPPC:DOPC:cholesterol vs DAPC, POPC, DPPC:cholesterol; different temperatures, e.g., 310 and $290 \mathrm{~K}$ vs 323 and $303 \mathrm{~K}$; different force fields, Slipids vs CHARMM27, Berger), several common features emerge:

(a) Cholesterol flip-flop occurs on the sub-millisecond time scale.

(b) Cholesterol flip-flop rate depends on the bilayer order; flip-flop is facilitated in bilayers with higher fluidity and lower rigidity. Two observations in our work support this point: lowering the temperature from 310 to $290 \mathrm{~K}$ increased the packing of lipid tails and reduced the flip-flop rate by 1 order of magnitude (Table 1); cholesterol flip-flop is $>7$ times faster in a DOPC-enriched local environment, compared with the DOPC depleted local environment (Figure 3, Figures S2 and S3). Earlier simulations also showed that lower cholesterol concentration $^{11,20}$ and polyunsaturated lipids ${ }^{24,25}$ facilitated cholesterol flip-flop. For instance, the flip-flop rate in DAPC was 10-1000 times higher than in POPC and DPPC bilayers; ${ }^{20,24}$ increasing the cholesterol concentration in DPPC membrane from 0 to $40 \%$ (probably leading to a phase transition from $\mathrm{L} \alpha$ to Lo phase) reduced the flip-flop rate by $\sim 1000$ times. ${ }^{47}$ Recent neutron scattering research ${ }^{48}$ also found a systematic decrease of cholesterol flip-flop rate with increasing saturation of the lipid tails in bilayers, albeit at a significantly longer flip-flop half-time (ranges from tens to hundreds of minutes), which is notably different from the time scale obtained by simulations and some other experiments. ${ }^{49-51}$

In addition, simulations in both this work and literature revealed larger cholesterol tilt angles in membranes with lower rigidity/order (compare Figure $2 \mathrm{~A}$ and Jo et al. ${ }^{24}$ and Marrink et al.'s ${ }^{25}$ work, where cholesterol tilt angle was calculated in bilayers with varying degrees of unsaturation). We also found a strong correlation between cholesterol tilt angle and the order parameter of its local environment, consistent with Aitonnemi et al.'s work. ${ }^{46}$ Based on these observations, we propose that less ordered domains/clusters apply less restriction on cholesterol orientation in bilayer and, therefore, allow larger tilt angle fluctuation and more flexible cholesterol orientation, which may facilitate cholesterol flip-flop.

The main finding of our simulations is that the local environment (specifically the first coordination shell of lipids) has a significant influence on cholesterol flip-flop rate, regardless of whether the membrane exhibits a stable phase separation or not (Figure 3B). The effect of local environment on flip-flop rate is as significant as the changes in temperature $(\sim 10$ times difference between 310 and $290 \mathrm{~K},>7$ times difference in DOPC-enriched and depleted environments). Lipid lateral organization in the plasma membrane is likely heterogeneous, whether lipid rafts exist or not; ${ }^{52}$ therefore, flip-flop rates in domains with different order may differ dramatically. As both moving out of and into a leaflet is facilitated in less ordered local environments, flip-flop might be promoted in bilayers with registered Ld domains and is likely to be much slower in bilayers with antiregistered domains. ${ }^{53}$ This effect should be more pronounced in bilayers with significant difference in order of coexisting domains. It is in debate whether cholesterol flip-flop promotes domain registration in phase separated membranes. $^{54-56}$ In addition, it is believed that the extracellular leaflet is more rigid than the cytosolic leaflet as the outer leaflet is more enriched in saturated lipid tails. ${ }^{6,57}$ If this is the case, the outer leaflet puts a limit on flip-flop rate, as overlap of disordered domains is essential for fast flip-flop (Figure S3). Another 
biological consequence is that flip-flop rates in cells with different plasma membrane composition may differ. Submillisecond time scale of passive cholesterol flip-flop implies that the distribution of cholesterol in the two leaflets reaches equilibrium quickly. ${ }^{11}$ Therefore, cholesterol flip-flop can be a mechanism that balances the area and stress of the two leaflets.

\section{CONCLUSION}

We estimated cholesterol flip-flop rate by counting spontaneous flip-flop events in a ternary mixture in atomistic simulations. The flip-flop rate lies on the (sub)millisecond time scale; fast cholesterol flip-flop thus allows quick equilibration of its distribution in the two leaflets of the plasma membrane. We found cholesterol flip-flop rate depends strongly on the local environment. The more disordered DOPC-enriched environment increases flip-flop rate by an order of magnitude, compared to the DPPC-enriched environment. We also described the atomic details of cholesterol flip-flop process and found a varying cholesterol orientation in the bilayer center.

\section{ASSOCIATED CONTENT}

\section{S Supporting Information}

The Supporting Information is available free of charge on the ACS Publications website at DOI: 10.1021/acs.jctc.8b00933.

DPPC-DOPC radial distribution function, histograms of DPPC enrichment values in the local environment of cholesterol, cholesterol flip-flop rate as a function of DPPC enrichment values, and overlap of disordered clusters facilitating cholesterol flip-flop (PDF)

Summary of all cholesterol flip-flop events at 310 and 290 $\mathrm{K}$, averages of lipid (DPPC, DOPC, or cholesterol) enrichment values in the local environment of cholesterol (XLSX)

\section{AUTHOR INFORMATION}

\section{Corresponding Author}

*E-mail: tieleman@ucalgary.ca.

\section{ORCID $\odot$}

Ruo-Xu Gu: 0000-0003-2587-0232

\section{Peter Tieleman: 0000-0001-5507-0688}

\section{Present Address}

${ }^{\dagger}$ Theoretical and Computational Biophysics Department, MaxPlanck Institute for Biophysical Chemistry, Am Faßberg 11, 37077 Göttingen, Germany.

\section{Notes}

The authors declare no competing financial interest.

\section{ACKNOWLEDGMENTS}

Work in D.P.T.'s group was supported by the Natural Sciences and Engineering Research Council of Canada. R.-X.G. was supported by postdoctoral fellowships from Alberta Innovates Health Solutions and the Canadian Institutes of Health Research (Funding Reference No. MFE-140949). D.P.T. holds the AITF Strategic Chair in (Bio)Molecular Simulation. Simulations were run on Compute Canada machines, supported by the Canada Foundation for Innovation and partners. This work was undertaken, in part, thanks to funding from the Canada Research Chairs program.

\section{REFERENCES}

(1) Contreras, F. X.; Sánchez-Magraner, L.; Alonso, A.; Goñi, F. M. Transbilayer (Flip-Flop) Lipid Motion and Lipid Scrambling in Membranes. FEBS Lett. 2010, 584, 1779-1786.

(2) Ikeda, M.; Kihara, A.; Igarashi, Y. Lipid Asymmetry of the Eukaryotic Plasma Membrane: Functions and Related Enzymes. Biol. Pharm. Bull. 2006, 29, 1542-1546.

(3) Kobayashi, T.; Menon, A. K. Transbilayer Lipid Asymmetry. Curr. Biol. 2018, 28, R386-R391.

(4) Fadeel, B.; Xue, D. The Ins and Outs of Phospholipid Asymmetry in the Plasma Membrane: Roles in Health and Disease. Crit. Rev. Biochem. Mol. Biol. 2009, 44, 264-277.

(5) van Meer, G.; Voelker, D. R.; Feigenson, G. W. Membrane Lipids: Where They Are and How They Behave. Nat. Rev. Mol. Cell Biol. 2008, $9,112-124$

(6) Steck, T. L.; Lange, Y. Transverse Distribution of Plasma Membrane Bilayer Cholesterol: Picking Sides. Traffic 2018, 19, 750760.

(7) Liu, S. L.; Sheng, R.; Jung, J. H.; Wang, L.; Stec, E.; O'Connor, M. J.; Song, S.; Bikkavilli, R. K.; Winn, R. A.; Lee, D.; Baek, K.; Ueda, K.; Levitan, I.; Kim, K. P.; Cho, W. Orthogonal Lipid Sensors Identify Transbilayer Asymmetry of Plasma Membrane Cholesterol. Nat. Chem. Biol. 2017, 13, 268-274.

(8) de Kruijff, B.; van den Besselaar, A. M.; Cullis, P. R.; van den Bosch, H.; van Deenen, L. L. Evidence for Isotropic Motion of Phospholipids in Liver Microsomal Membranes. A 31P NMR Study. Biochim. Biophys. Acta, Biomembr. 1978, 514, 1-8.

(9) Wimley, W. C.; Thompson, T. E. Exchange and Flip-Flop of Dimyristoylphosphatidylcholine in Liquid-Crystalline, Gel, and TwoComponent, Two-Phase Large Unilamellar Vesicles. Biochemistry 1990, 29, 1296-1303.

(10) Zachowski, A.; Devaux, P. F. Transmembrane Movements of Lipids. Experientia 1990, 46, 644-656.

(11) Bennett, W. F. D.; MacCallum, J. L.; Hinner, M. J.; Marrink, S. J.; Tieleman, D. P. Molecular View of Cholesterol Flip-Flop and Chemical Potential in Different Membrane Environments. J. Am. Chem. Soc. 2009, 131, 12714-12720.

(12) Bruckner, R. J.; Mansy, S. S.; Ricardo, A.; Mahadevan, L.; Szostak, J. W. Flip-Flop-Induced Relaxation of Bending Energy: Implications for Membrane Remodeling. Biophys. J. 2009, 97, 3113-3122.

(13) Steck, T. L.; Ye, J.; Lange, Y. Probing Red Cell Membrane Cholesterol Movement with Cyclodextrin. Biophys. J. 2002, 83, 2118 2125.

(14) Leventis, R.; Silvius, J. R. Use of Cyclodextrins to Monitor Transbilayer Movement and Differential Lipid Affinities of Cholesterol. Biophys. J. 2001, 81, 2257-2267.

(15) Garg, S.; Porcar, L.; Woodka, A. C.; Butler, P. D.; Perez-Salas, U. Noninvasive Neutron Scattering Measurements Reveal Slower Cholesterol Transport in Model Lipid Membranes. Biophys. J. 2011, 101, 370-377.

(16) Schroeder, F.; Jefferson, J. R.; Kier, A. B.; Knittel, J.; Scallen, T. J.; Wood, W. G.; Hapala, I. Membrane Cholesterol Dynamics: Cholesterol Domains and Kinetic Pools. Exp. Biol. Med. 1991, 196, 235-252.

(17) Rodrigueza, W. V.; Klimuk, S. K.; Kitson, C. N.; Wheeler, J. J.; Hope, M. J. Transbilayer Movement and Net Flux of Cholesterol and Cholesterol Sulfate between Liposomal Membranes. Biochemistry 1995, 34, 6208-6217.

(18) Bacia, K.; Schwille, P.; Kurzchalia, T. Sterol Structure Determines the Separation of Phases and the Curvature of the Liquid-Ordered Phase in Model Membranes. Proc. Natl. Acad. Sci. U. S. A. 2005, 102, 3272-3277.

(19) Yu, T.; Zhou, G.; Hu, X.; Ye, S. Transport and Organization of Cholesterol in a Planar Solid-Supported Lipid Bilayer Depend on the Phospholipid Flip-Flop Rate. Langmuir 2016, 32, 11681-11689.

(20) Bennett, W. F. D.; Tieleman, D. P. Molecular Simulation of Rapid Translocation of Cholesterol, Diacylglycerol, and Ceramide in Model Raft and Nonraft Membranes. J. Lipid Res. 2012, 53, 421-429. 
(21) Parisio, G.; Sperotto, M. M.; Ferrarini, A. Flip-Flop of Steroids in Phospholipid Bilayers: Effects of the Chemical Structure on Transbilayer Diffusion. J. Am. Chem. Soc. 2012, 134, 12198-12208.

(22) Parisio, G.; Ferrarini, A.; Sperotto, M. M. Model Studies of Lipid Flip-Flop in Membranes. Int. J. Adv. Eng. Sci. Appl. Math. 2016, 8, 134146.

(23) Choubey, A.; Kalia, R. K.; Malmstadt, N.; Nakano, A.; Vashishta, P. Cholesterol Translocation in a Phospholipid Membrane. Biophys. J. 2013, 104, 2429-2436.

(24) Jo, S.; Rui, H.; Lim, J. B.; Klauda, J. B.; Im, W. Cholesterol FlipFlop: Insights From Free Energy Simulation Studies. J. Phys. Chem. B 2010, 114, 13342-13348.

(25) Marrink, S. J.; de Vries, A. H.; Harroun, T. A.; Katsaras, J.; Wassall, S. R. Cholesterol Shows Preference for the Interior of Polyunsaturated Lipid Membranes. J. Am. Chem. Soc. 2008, 130, 1011.

(26) Levental, I.; Veatch, S. L. The Continuing Mystery of Lipid Rafts. J. Mol. Biol. 2016, 428, 4749-4764.

(27) Sezgin, E.; Levental, I.; Mayor, S.; Eggeling, C. The Mystery of Membrane Organization: Composition, Regulation and Roles of Lipid Rafts. Nat. Rev. Mol. Cell Biol. 2017, 18, 361-374.

(28) Honigmann, A.; Mueller, V.; Ta, H.; Schoenle, A.; Sezgin, E.; Hell, S. W.; Eggeling, C. Scanning STED-FCS Reveals Spatiotemporal Heterogeneity of Lipid Interaction in the Plasma Membrane of Living Cells. Nat. Commun. 2014, 5, 5412.

(29) Nickels, J. D.; Chatterjee, S.; Stanley, C. B.; Qian, S.; Cheng, X.; Myles, D. A. A.; Standaert, R. F.; Elkins, J. G.; Katsaras, J. The in Vivo Structure of Biological Membranes and Evidence for Lipid Domains. PLoS Biol. 2017, 15, No. e2002214.

(30) Jorgensen, W. L.; Chandrasekhar, J.; Madura, J. D.; Impey, R. W.; Klein, M. L. Comparison of Simple Potential Functions for Simulating Liquid Water. J. Chem. Phys. 1983, 79, 926-935.

(31) Pronk, S.; Páll, S.; Schulz, R.; Larsson, P.; Bjelkmar, P.; Apostolov, R.; Shirts, M. R.; Smith, J. C.; Kasson, P. M.; van der Spoel, D.; Hess, B.; Lindahl, E. GROMACS 4.5: A High-Throughput and Highly Parallel Open Source Molecular Simulation Toolkit. Bioinformatics 2013, 29, 845-854.

(32) Jämbeck, J. P. M.; Lyubartsev, A. P. Derivation and Systematic Validation of a Refined All-Atom Force Field for Phosphatidylcholine Lipids. J. Phys. Chem. B 2012, 116, 3164-3179.

(33) Jämbeck, J. P. M.; Lyubartsev, A. P. Another Piece of the Membrane Puzzle: Extending Slipids Further. J. Chem. Theory Comput. 2013, 9, 774-784.

(34) Jämbeck, J. P. M.; Lyubartsev, A. P. An Extension and Further Validation of an All-Atomistic Force Field for Biological Membranes. J. Chem. Theory Comput. 2012, 8, 2938-2948.

(35) Darden, T.; York, D.; Pedersen, L. Particle Mesh Ewald: An N $\log (\mathrm{N})$ Method for Ewald Sums in Large Systems. J. Chem. Phys. 1993, 98, 10089-10092.

(36) Essmann, U.; Perera, L.; Berkowitz, M. L.; Darden, T.; Lee, H.; Pedersen, L. G. A Smooth Particle Mesh Ewald Method. J. Chem. Phys. 1995, 103, 8577-8592.

(37) Bussi, G.; Donadio, D.; Parrinello, M. Canonical Sampling Through Velocity Rescaling. J. Chem. Phys. 2007, 126, 014101.

(38) Parrinello, M.; Rahman, A. Polymorphic Transitions in Single Crystals: A New Molecular Dynamics Method. J. Appl. Phys. 1981, 52, 7182-7190.

(39) Nose, S.; Klein, M. L. Constant Pressure Molecular Dynamics for Molecular Systems. Mol. Phys. 1983, 50, 1055-1076.

(40) Hess, B.; Bekker, H.; Berendsen, H. J. C.; Fraaije, J. G. E. M. LINCS: A Linear Constraint Solver for Molecular Simulations. J. Comput. Chem. 1997, 18, 1463-1472.

(41) Rozmanov, D.; Tieleman, D. P. Bio. B-Gen: An Initial System Generator for Biological Molecular Simulations. Biophys. J. 2015, 108, 158 a.

(42) Bio. B-Gen: Initial system (box) generator for biological molecular simulations. https://sourceforge.net/projects/biobgen/ (accessed Aug. 2, 2015).
(43) Sodt, A. J.; Sandar, M. L.; Gawrisch, K.; Pastor, R. W.; Lyman, E. The Molecular Structure of the Liquid Ordered Phase of Lipid Bilayers. J. Am. Chem. Soc. 2014, 136, 725-732.

(44) Michaud-Agrawal, N.; Denning, E. J.; Woolf, T. B.; Beckstein, O. MDAnalysis: A Toolkit for the Analysis of Molecular Dynamics Simulations. J. Comput. Chem. 2011, 32, 2319-2327.

(45) Baoukina, S.; Rozmanov, D.; Tieleman, D. P. Composition Fluctuations in Lipid Bilayers. Biophys. J. 2017, 113, 2750-2761.

(46) Aittoniemi, J.; Róg, T.; Niemelä, P.; Pasenkiewicz-Gierula, M.; Karttunen, M.; Vattulainen, I. Tilt: Major Factor in Sterols' Ordering Capability in Membranes. J. Phys. Chem. B 2006, 110, 25562-25564.

(47) Bennett, W. F. D.; MacCallum, J. L.; Tieleman, D. P. Thermodynamic Analysis of the Effect of Cholesterol on Dipalmitoylphosphatidylcholine Lipid Membranes. J. Am. Chem. Soc. 2009, 131, 1972-1978.

(48) Breidigan, J. M.; Krzyzanowski, N.; Liu, Y.; Porcar, L.; PerezSalas, U. Influence of the Membrane Environment on Cholesterol Transfer. J. Lipid Res. 2017, 58, 2255-2263.

(49) Atkovska, K.; Klingler, J.; Oberwinkler, J.; Keller, S.; Hub, J. S. Rationalizing Steroid Interactions with Lipid Membranes: Conformations, Partitioning, and Kinetics. ACS Cent. Sci. 2018, 4, 1155-1165.

(50) Hamilton, J. A. Fast Flip-Flop of Cholesterol and Fatty Acids in Membranes: Implications for Membrane Transport Proteins. Curr. Opin. Lipidol. 2003, 14, 263-271.

(51) Backer, J. M.; Dawidowicz, E. A. The Rapid Transmembrane Movement of Cholesterol in Small Unilamellar Vesicles. Biochim. Biophys. Acta, Biomembr. 1979, 551, 260-270.

(52) Lingwood, D.; Simons, K. Lipid Rafts As a MembraneOrganizing Principle. Science 2010, 327, 46-50.

(53) Nickels, J. D.; Smith, J. C.; Cheng, X. Lateral Organization, Bilayer Asymmetry, and Inter-Leaflet Coupling of Biological Membranes. Chem. Phys. Lipids 2015, 192, 87-99.

(54) Putzel, G. G.; Uline, M. J.; Szleifer, I.; Schick, M. Interleaflet Coupling and Domain Registry in Phase-Separated Lipid Bilayers. Biophys. J. 2011, 100, 996-1004.

(55) May, S. Trans-Monolayer Coupling of Fluid Domains in Lipid Bilayers. Soft Matter 2009, 5, 3148-3156.

(56) Simons, K.; Toomre, D. Lipid Rafts and Signal Transduction. Nat. Rev. Mol. Cell Biol. 2000, 1, 31-39.

(57) Ingólfsson, H. I.; Melo, M. N.; van Eerden, F. J.; Arnarez, C.; López, C. A.; Wassenaar, T. A.; Periole, X.; de Vries, A. H.; Tieleman, D. P.; Marrink, S. J. Lipid Organization of the Plasma Membrane. J. Am. Chem. Soc. 2014, 136, 14554-14559. 